\title{
DERIVED FUNCTORS OF THE TORSION FUNCTOR AND LOCAL COHOMOLOGY OF NONCOMMUTATIVE RINGS
}

\author{
JONATHAN S. GOLAN and JACQUES RAYNAUD
}

(Received 5 January 1982)

Communicated by R. Lidl

\begin{abstract}
Let $R$ be an associative ring which is not necessarily commutative. For any torsion theory $\tau$ on the category of left $R$-modules and for any nonnegative integer $n$ we define and study the notion of the $n$th local cohomology functor with respect to $\tau$. For suitably nice rings a bound for the nonvanishing of these functors is given in terms of the $\tau$-dimension of the modules.
\end{abstract}

1980 Mathematics subject classification (Amer. Math. Soc.): primary 16A08, 16A63, 18E25, 18G10; secondary 13D03, 16A55, 18E40.

The right derived functors of the torsion functor determined by an arbitrary torsion theory on a module category were first studied by Dickson [7]. The relation between torsion theories and local cohomology was first considered by Suominen [21] for the special case of categories of sheaves. For module categories over a commutative ring the basic results were obtained by Cahen [6] and these have recently been extended by Albu and Nastasescu [1,2] and by Bijan-Zadeh [5]. Our purpose here is to show how similar results can be obtained for categories of modules over noncommutative rings.

Throughout the following, $R$ will denote an arbitrary associative (but not necessarily commutative) ring with unit element 1 . The category of unitary left $R$-modules will be denoted by $R$-mod. Morphisms in $R$-mod will be written as acting on the right. All other functions will be written as acting on the left. If $M$ is a left $R$-module then the injective hull of $M$ will be denoted by $E(M)$.

The complete brouwerian lattice of all hereditary torsion theories defined on $R$-mod will be denoted by $R$-tors. Notation and terminology concerning such

(c) 1983 Australian Mathematical Society $0263-6115 / 83 \$ A 2.00+0.00$ 
theories will follow [8]. In particular, if $\tau \in R$-tors we denote the $\tau$-torsion endofunctor of $R$-mod by $T_{\tau}(-)$ and the $\tau$-localization endofunctor of $R$-mod by $Q_{\tau}(-)$. If $M$ is a left $R$-module then the canonical $R$-homomorphism from $M$ to $Q_{\tau}(M)$ will be denoted by $\lambda_{M}^{\tau}$ and not, as in [8], by $\hat{\tau}_{M}$. The localization of the ring $R$ at $\tau$ will be denoted by $R_{\tau}$. The $\tau$-injective hull of a left $R$-module $M$ will be denoted by $E_{\tau}(M)$. A submodule $N$ of a left $R$-module $M$ is said to be $\tau$-dense in $M$ if and only if $M / N$ is a $\tau$-torsion left $R$-module.

If $M$ is a left $R$-module then the meet of all torsion theories relative to which $M$ is torsion will be denoted by $\xi(M)$ and the join of all torsion theories relative to which $M$ is torsionfree will be denoted by $\chi(M)$. Then $\xi=\xi(0)$ is the unique minimal element of $R$-tors and $\chi=\chi(0)$ is the unique maximal element of $R$-tors.

A nonzero $\tau$-torsionfree left $R$-module $M$ is said to be $\tau$-cocritical if and only if every nonzero submodule of $M$ is $\tau$-dense in it. Such modules are necessarily uniform. A left $R$-module is said to be cocritical if and only if it is $\tau$-cocritical for some torsion theory $\tau$. A torsion theory of the form $\chi(M)$ for some cocritical left $R$-module $M$ is said to be prime. The set of all prime torsion theories in $R$-tors is denoted by $R$-sp. Any theory theory $\tau \in R$-tors partitions $R$-sp into two disjoint parts:

$$
\mathbf{P}(\tau)=\{\pi \in R-\mathrm{sp} \mid \pi \geqslant \tau\} \quad \text { and } \quad \mathrm{V}(\tau)=\{\pi \in R-\mathrm{sp} \mid \pi \neq \tau\} .
$$

If $M$ is a left $R$-module then the set of associated primes of $M$, denoted by ass $(M)$, is the set of all primes in $R$-sp of the form $\chi(N)$, where $N$ is a cocritical submodule of $M$. The ring $R$ is said to be left definite if and only if $\operatorname{ass}(M) \neq \varnothing$ for any nonzero left $R$-module $M$. Left noetherian rings are easily seen to be left definite. If $R$ is left definite then $\tau=\wedge \mathbf{P}(\tau)$ for any torsion theory $\tau$ in $R$-tors other than $\chi$. (In fact, this relation holds for an even larger class of rings, which need not concern us here.)

For any nonempty subset $U$ of $R$-sp we can define a torsion theory $\delta(U)$ in $R$-tors by saying that a left $R$-module $M$ is $\delta(U)$-torsion if and only if the following conditions hold:

(i) every nonzero homomorphic image of $M$ has a cocritical submodule; and

(ii) if $N$ is a cocritical submodule of a nonzero homomorphic image of $M$ then $\chi(N) \in U$.

If $U \subseteq U^{\prime}$ are nonempty subsets of $R$-sp then it is clear that $\delta(U) \leqslant \delta\left(U^{\prime}\right)$. Then the ring $R$ is left definite if and only if for every torsion theory $\tau$ in $R$-tors there exists a subset $U$ of $R$-sp for which $\tau=\delta(U)$ [10, Proposition 2]. Indeed, if $R$ is left definite then for any subset $U$ of $R$-sp we also have $\delta(U)=\wedge[R$-sp $\backslash U]$ [17].

The support of a left $R$-module $M$, denoted by $\operatorname{supp}(M)$, consists of all those elements of $R$-sp relative to which $M$ is not torsion. If $R$ is a left definite ring and 
if $U$ is a nonempty subset of $R$-sp then a left $R$-module $M$ is $\delta\left(U_{0}\right)$-torsion if and only if $\operatorname{supp}(M) \subseteq U$.

Finally, a torsion theory $\tau$ in $R$-tors is said to be perfect if and only if every left $R_{\tau}$-module is $\tau$-torsionfree when considered as a left $R$-module.

\section{Local cohomology functors}

Let $\tau \in R$-tors. For any nonnegative integer $n$ we define the $n$th local cohomology functor with respect to $\tau$ to be the $n$th right derived functor $R^{n} T_{\tau}(-)$ of the $\tau$-torsion endofunctor of $R$-mod. In particular, we note that $R^{n} T_{\tau}(M)$ is a $\tau$-torsion left $R$-module for any left $R$-module $M$ and that $R^{0} T_{\tau}(-)$ equals $T_{\tau}(-)$ since the latter functor is always left exact. Moreover, the proof of Proposition 2.1 of [1] carries over to the noncommutative case and so we see that for any left $R$-module $M$ and for each nonegative integer $n$ there exists a natural isomorphism in the category of abelian groups between $R^{n} T_{\tau}(M)$ and $\lim \operatorname{Ext}_{R}^{n}(R / I, M)$, where the limit is taken over the idempotent filter of all $\tau$-dense left ideals of $R$. Moreover, if the ring $R$ is left noetherian and if $\left\{M_{i} \mid i \in \Omega\right\}$ is a directed system of left $R$-modules then for each nonnegative integer $n$ we have $\lim _{i \in \Omega} R^{n} T\left(M_{i}\right) \cong$ $R^{n} T_{\tau}\left(\lim M_{i}\right)$.

Let $\vec{M}$ be a nonzero left $R$-module having a minimal injective resolution

$$
0 \rightarrow M \rightarrow E_{0} \rightarrow E_{1} \rightarrow \cdots
$$

and for each $k \geqslant 0$ let $\chi_{k}(M)=\chi\left(E_{0} \oplus \cdots \oplus E_{k}\right)=\wedge_{i=0}^{k} \chi\left(E_{i}\right)$. Then a left $R$-module $N$ is $\chi_{k}(M)$-torsion if and only if $\operatorname{Ext}_{R}^{i}\left(N^{\prime}, M\right)=0$ for any [cyclic] submodule $N^{\prime}$ of $N$ and any $i \leqslant k$. See page 149 of [19] for details. For notational simplicity, we set $\chi_{-1}(M)=\chi$ for any left $R$-module $M$.

If $M$ is a left $R$-module, if $n$ is a nonnegative integer, and if $\tau \in R$-tors then we say that $M$ has $\tau$-dominant dimension equal to $n$ if and only if $\chi_{n-1}(M) \leqslant \tau$ and $\chi_{n}(M) \neq \tau$. In terms of the above minimal injective resolution of $M$, this is equivalent to saying that $E_{i}$ is $\tau$-torsionfree for all $i<n$, while $E_{n}$ is not $\tau$-torsionfree. We denote the $\tau$-dominant dimension of $M$ by $\tau$-dom.dim $(M)$. If $\tau$-dom.dim $(M) \neq n$ for any nonnegative integer $n$, we write $\tau$-dom.dim $(M)=\infty$. Dominant dimension has been extensively studied. See, for example, $[14,16,20]$.

(1.1) Example. A ring $R$ is said to be left local if and only if all simple left $R$-modules are isomorphic. Let $R$ be a left local ring and let $N$ be a simple left $R$-module. For any left $R$-module $M$, we see that $\xi(N)$-dom.dim $(M)=0$ if and only if $E(M)$ is not $\xi(N)$-torsionfree, that is, if and only if $\operatorname{Hom}_{R}(N, E(M)) \neq 0$. 
But this condition is equivalent to the condition that $E(M)$ (and hence $M$ ) have a submodule isomorphic to $N$. Thus we see that $\xi(N)$-dom.dim( $M)=0$ if and only if $\operatorname{soc}(M) \neq 0$.

(1.2) Proposition. If $\tau \in R$-tors and if $n$ is a natural number then the following conditions on a left $R$-module $M$ are equivalent:

(1) $\tau$-dom.dim $(M) \geqslant n$.

(2) $R^{i} T_{\tau}(M)=0$ for all $i<n$.

Proof. We will proceed by induction on $n$. In particular, we note that $\tau$-dom.dim $(M) \geqslant 1 \Leftrightarrow M$ is $\tau$-torsionfree $\Leftrightarrow R^{0} T_{\tau}(M)=0$. Now assume inductively that $n>1$ and that whenever $k<n$ we have $\tau$-dom.dim $\left(M^{\prime}\right) \geqslant k \Leftrightarrow$ $R^{i} T_{\tau}\left(M^{\prime}\right)=0$ for all $i<k$, this holding for any left $R$-module $M^{\prime}$. In particular, let $\bar{M}=E(M) / M$. Then

$$
\begin{aligned}
R^{i} T_{\tau}(M)=0 \text { for all } i<n \Leftrightarrow & M \text { is } \tau \text {-torsion free and } R^{i} T_{\tau}(\bar{M})=0 \\
& \text { for all } i<n-1 \\
\Leftrightarrow & M \text { is } \tau \text {-torsionfree and } \\
& \tau \text {-dom.dim }(\bar{M}) \geqslant n-1 \\
\Leftrightarrow & \tau \text {-dom.dim }(M) \geqslant n,
\end{aligned}
$$

and so we are done.

The commutative version of this theorem was proven in [6].

(1.3) COROllary. If $\tau \in R$-tors and if $M$ is a left $R$-module satisfying $\tau$ $\operatorname{dom} \cdot \operatorname{dim}(M) \geqslant n$ then for any $R$-monomorphism $\alpha: M \rightarrow M$ we have $\tau$ $\operatorname{dom} \cdot \operatorname{dim}(M / M \alpha) \geqslant n-1$.

Proof. By hypothesis we have an exact sequence $0 \rightarrow M \stackrel{\alpha}{\rightarrow} M \rightarrow M / M \alpha \rightarrow 0$ of left $R$-modules which induces a long exact sequence

$$
\cdots \rightarrow R^{i} T_{\tau}(M) \rightarrow R^{i} T_{\tau}(M / M \alpha) \rightarrow R^{i+1} T_{\tau}(M) \rightarrow \cdots .
$$

Since $R^{i} T_{\tau}(M)=0$ for all $i<n$ by Proposition 1.2, we have $R^{i} T_{\tau}(M / M \alpha)=0$ for all $i<n-1$ and so, by Proposition 1.2, $\tau$-dom.dim $(M / M \alpha) \geqslant n-1$.

We would now like to calculate $R^{i} T_{\tau}(M)$ for certain types of torsion theories $\tau$ and left $R$-modules $M$. Recall that a torsion theory $\tau \in R$-tors is stable if and only if the class of all $\tau$-torsion left $R$-modules is closed under taking injective hulls. The basic properties of stable torsion theories are summarized in [8]. In particular, if $R$ is a commutative noetherian ring then every element of $R$-tors is stable. 
For any torsion theory $\tau \in R$-tors and for any $\tau$-torsion left $R$-module $M$ we have $R^{1} T_{\tau}(M)=0[7$, Lemma 2]. For stable torsion theories this result can be further extended.

(1.4) Proposition. If $\tau \in R$-tors is stable and if $M$ is a $\tau$-torsion left $R$-module then $R^{i} T_{\tau}(M)=0$ for all $i>0$.

Proof. Let $0 \rightarrow M \rightarrow E_{0} \rightarrow E_{1} \rightarrow \cdots$ be a minimal injective resolution of $M$. Since $M$ is $\tau$-torsion and since $\tau$ is stable, we see that each $E_{i}$ is $\tau$-torsion and so the complex $0 \rightarrow T_{\tau}\left(E_{0}\right) \rightarrow T_{r}\left(E_{1}\right) \rightarrow \cdots$ is exact at $T_{\tau}\left(E_{i}\right)$ for all $i>0$, which is what we need to show.

(1.5) COROLlaRy. If $\tau \in R$-tors is stable and if $M$ is a left $R$-module then $R^{i} T_{\tau}(M) \cong R^{i} T_{\tau}\left(M / T_{\tau}(M)\right)$ for all $i>0$.

Proof. The exact sequence $0 \rightarrow T_{\tau}(M) \rightarrow M \rightarrow M / T_{\tau}(M) \rightarrow 0$ induces a long exact sequence

$$
0 \rightarrow T_{\tau}\left(T_{\tau}(M)\right) \rightarrow T_{\tau}(M) \rightarrow T_{\tau}\left(M / T_{\tau}(M)\right) \rightarrow R^{1} T_{\tau}\left(T_{\tau}(M)\right) \rightarrow \cdots
$$

in which, by Proposition 1.4, we know that $R^{i} T_{\tau}\left(T_{\tau}(M)\right)=0$ for all $i>0$. From this the result follows immediately.

The following result was first established for commutative rings by Cahen [6].

(1.6) Proposition. If $\tau \in R$-tors is stable and if $M$ is a left $R$-module then $R^{1} T_{\tau}(M) \cong \operatorname{coker}\left(\lambda_{M}^{\tau}\right)$.

Proof. Set $K_{\tau}=\operatorname{coker}\left(\lambda_{M}^{\tau}\right)$. Then the short exact sequence

$$
0 \rightarrow M / T_{\tau}(M) \rightarrow Q_{\tau}(M) \rightarrow K_{\tau} \rightarrow 0
$$

gives rise to a long exact sequence

$$
0 \rightarrow T_{\tau}\left(K_{\tau}\right) \rightarrow R^{1} T_{\tau}\left(M / T_{\tau}(M)\right) \rightarrow R^{1} T_{\tau}\left(Q_{\tau}(M)\right) \rightarrow \cdots .
$$

Since $Q_{\tau}(M)$ is $\tau$-torsionfree and $\tau$-injective, we see that $\tau$-dom.dim $\left(Q_{\tau}(M)\right) \geqslant 2$ [20] and so $R^{1} T_{\tau}\left(Q_{\tau}(M)\right)=0$. Moreover, $K_{\tau}$ is $\tau$-torsion by construction of $Q_{\tau}(M)$ and so $T_{\tau}\left(K_{\tau}\right)=K_{\tau}$. Therefore, by Corollary $1.5, K_{\tau} \cong R^{1} T_{\tau}\left(M / T_{\tau}(M)\right)$ $\cong R^{1} T_{\tau}(M)$.

(1.7) Proposition. If $\tau \in R$-tors is stable and if $M$ is a nonzero $\tau$-dense submodule of its injective hull then $R^{i} T_{\tau}(M)=0$ for all $i>1$. 
Proof. The short exact sequence

$$
0 \rightarrow M \rightarrow E(M) \rightarrow E(M) / M \rightarrow 0
$$

gives rise to a long exact sequence

$$
\begin{aligned}
\cdots & \rightarrow R^{1} T_{\tau}(E(M) / M) \rightarrow R^{2} T_{\tau}(M) \\
& \rightarrow R^{2} T_{\tau}(E(M)) \rightarrow R^{2} T_{\tau}(E(M) / M) \rightarrow \cdots .
\end{aligned}
$$

By Proposition 1.4, we know that $R^{i} T_{\tau}(E(M) / M)=0$ for all $i>0$. Moreover, since, as abelian groups, we have $R^{i} T_{\tau}(E(M)) \cong \lim \operatorname{Ext}_{R}^{i}(R / I, E(M)$ ) (where the limit is taken over the filter of all $\tau$-dense left ideals $I$ of $R$ ) and since $E(M)$ is injective, we see that $R^{i} T_{\tau}(E(M))=0$ for all $i>0$. Therefore $R^{i} T_{\tau}(M)=0$ for all $i>1$.

(1.8) Proposition. If $\tau \in R$-tors is stable and if $M$ is a $\tau$-torsionfree left $R$-module then

(1) $R^{0} T_{\tau}(M)=0$

(2) $R^{1} T_{\tau}(M) \cong E_{\tau}(M) / M$;

(3) $R^{i} T_{\tau}(M) \cong R^{i} T_{\tau}\left(E_{\tau}(M)\right)$ for all $i>1$.

Proof. (1) follows directly from the fact that $R^{0} T_{\tau}(M)=T_{\tau}(M)$. Moreover, the short exact sequence

$$
0 \rightarrow M \rightarrow E_{\tau}(M) \rightarrow E_{\tau}(M) / M \rightarrow 0
$$

yields a long exact sequence

$$
\begin{aligned}
0 \rightarrow R^{0} T_{\tau}(M) \rightarrow R^{0} T_{\tau}( & \left.E_{\tau}(M)\right) \rightarrow R^{0} T_{\tau}\left(E_{\tau}(M) / M\right) \rightarrow R^{1} T_{\tau}(M) \\
& \rightarrow R^{1} T_{\tau}\left(E_{\tau}(M)\right) \rightarrow R^{1} T_{\tau}\left(E_{\tau}(M) / M\right) \rightarrow R^{2} T_{\tau}(M) \rightarrow \cdots
\end{aligned}
$$

in which $R^{0} T_{\tau}(M)=R^{0} T_{\tau}\left(E_{\tau}(M)\right)=0$ by (1) and $R^{\prime} T_{\tau}\left(E_{\tau}(M) / M\right)=0$ for all $i>0$ by Proposition 1.4. In particular, this implies (3). Finally, (2) follows directly from Proposition 1.6.

(1.9) Proposition. Let $\tau \in R$-tors and let $M$ be a left $R$-module having minimal injective resolution

$$
0 \rightarrow M \rightarrow E_{0} \stackrel{\alpha_{0}}{\rightarrow} E_{1} \stackrel{\alpha_{1}}{\rightarrow} E_{2} \rightarrow \cdots .
$$

If $M_{i}=\operatorname{ker}\left(\alpha_{i}\right)$ for all $i \geqslant 0$ then $R^{k} T_{\tau}\left(M_{i}\right) \cong R^{k-1} T_{\tau}\left(M_{i+1}\right)$ for any $k \geqslant 2$. Moreover, if $R^{0} T_{\tau}\left(M_{i}\right)=0$ then $R^{1} T_{\tau}\left(M_{i}\right) \cong R^{0} T_{\tau}\left(M_{i+1}\right)$. 
Proof. From the exact sequence $0 \rightarrow M_{i} \rightarrow E_{i} \rightarrow M_{i+1} \rightarrow 0$ we obtain the long exact sequence

$$
\begin{aligned}
0 & \rightarrow R^{0} T_{\tau}\left(M_{i}\right) \rightarrow R^{0} T_{\tau}\left(E_{i}\right) \rightarrow R^{0} T_{\tau}\left(M_{i+1}\right) \rightarrow R^{\mathrm{I}} T_{\tau}\left(M_{i}\right) \\
& \rightarrow R^{\mathrm{I}} T_{\tau}\left(E_{i}\right) \rightarrow R^{\mathrm{l}} T_{\tau}\left(M_{i+1}\right) \rightarrow \cdots \rightarrow R^{k-1} T_{\tau}\left(E_{i}\right) \\
& \rightarrow R^{k-1} T_{\tau}\left(M_{i+1}\right) \rightarrow R^{k} T_{\tau}\left(M_{i}\right) \rightarrow R^{k} T_{\tau}\left(E_{i}\right) \rightarrow \cdots
\end{aligned}
$$

from which we obtain the desired result since for all $k>0$ we have $R^{k} T_{\tau}\left(E_{i}\right)=0$ by the injectivity of $E_{i}$.

As an immediate consequence of Proposition 1.9 we see that if $\tau \in R$-tors and if $M$ is a left $R$-module then for all positive integers $k$ and $h$ we have $R^{k+h} T_{\tau}(M)$ $\cong R^{h} T_{\tau}\left(M_{k}\right)$, where $M_{k}$ is defined as in the proof of Proposition 1.9.

(1.10) Proposition. Let $\tau \leqslant \sigma$ be stable torsion theories in R-tors. For any nonnegative integer $k$ and any left $R$-module $M$ the condition

(1) $R^{i} T_{\sigma}(M)=0$ for all $i \leqslant k$

implies

(2) $R^{i} T_{\tau}(M)=0$ for all $i \leqslant k$.

Proof. If $k=0$ then for any left $R$-module $M$ we have $R^{0} T_{\sigma}(M)=T_{\sigma}(M) \supseteq$ $T_{\tau}(M)=R^{0} T_{\tau}(M)$ and so the result is immediate. Next assume that $k=1$. If $M$ is a left $R$-module satisfying (1) then, in particular, $M$ is $\sigma$-torsionfree and hence $\tau$-torsionfree. Therefore, by Proposition 1.8, we have $R^{l} T_{\tau}(M) \cong E_{\tau}(M) / M \subseteq$ $E_{\sigma}(M) / M \cong R^{1} T_{\sigma}(M)$ and so $R^{1} T_{\sigma}(M)=0$ implies that $R^{1} T_{\tau}(M)=0$.

Now assume inductively that $k>1$ and that any left $R$-module $M$ satisfying $R^{i} T_{\sigma}(M)=0$ for all $i \leqslant k-1$ also satisfies $R^{i} T_{\tau}(M)=0$ for all $i \leqslant k-1$. By Proposition 1.9 we have $R^{i} T_{\sigma}(M) \cong R^{k-1} T_{\sigma}(E(M) / M)$ and $R^{k} T_{r}(M) \cong$ $R^{k-1} T_{\tau}(E(M) / M)$. By assumption, $0=R^{i} T_{\sigma}(M) \cong R^{i-1} T_{\sigma}(E(M) / M)$ for all $0<i \leqslant k$ and so, by the induction hypothesis, we see that $R^{i-1} T_{\tau}(E(M) / M)=0$ for all $0<i \leqslant k$. Therefore $R^{i} T_{\tau}(M)=0$ for all $0<i \leqslant k$. Moreover, we have already seen that $R^{0} T_{\mathrm{o}}(M)=0$ implies that $R^{0} T_{\tau}(M)=0$ as well.

A torsion theory $\tau \in R$-tors is exact if and only if the localization functor $Q_{\mathrm{T}}(-): R-\bmod \rightarrow R-\bmod$ is exact. See Section 16 of [8] for details about such torsion theories.

(1.11) Proposition. The following conditions on a torsion theory $\tau \in R$-tors are equivalent:

(1) $\tau$ is exact;

(2) If $M$ is a $\tau$-torsionfree $\tau$-injective left $R$-module then $R^{i} T_{\tau}(M)=0$ for all $i \geqslant 0$. 
Proof. (1) $\Rightarrow(2)$ : Let

$$
0 \rightarrow M \rightarrow E_{0} \stackrel{\alpha_{0}}{\rightarrow} E_{1} \stackrel{\alpha_{1}}{\rightarrow} E_{2} \rightarrow \cdots
$$

be a minimal injective resolution of $M$. By repeated application of Proposition 16.1 of [8] we see that $E_{i} / \operatorname{ker}\left(\alpha_{i}\right)$ is $\tau$-torsionfree and $\tau$-injective for all $i \geqslant 0$ and hence $E_{i}$ is $\tau$-torsionfree for all such $i$. This proves that $R^{i} T_{\tau}(M)=0$ for all $i \geqslant 0$.

(2) $\Rightarrow(1)$ : If $M$ is a left $R$-module which is $\tau$-torsionfree and $\tau$-injective then $T_{\tau}(E(M) / M)=E_{\tau}(M) / M=0$. Let (*), as above, be a minimal injective resolution of $M$. Then $E_{0} / M$ is $\tau$-torsionfree and hence so is $E_{1}$. Therefore $R^{2} T_{\tau}(M)=$ $\operatorname{ker}\left(T_{\tau}\left(\alpha_{2}\right)\right)=T_{\tau}\left(\operatorname{ker}\left(\alpha_{2}\right)\right)$. By hypothesis, $\operatorname{ker}\left(\alpha_{2}\right) \cong E\left(\operatorname{ker}\left(\alpha_{1}\right)\right) / \operatorname{ker}\left(\alpha_{1}\right)$ is $\tau$ torsionfree. Therefore $\operatorname{ker}\left(\alpha_{1}\right)$ is $\tau$-injective. But it is also $\tau$-torsionfree and so by Proposition 16.1 of [8] we see that $Q_{\tau}(-)$ is exact and so the torsion theory $\tau$ is exact.

(1.12) CoROllaRY. If $\tau \in R$-tors is exact and stable and if $M$ is a $\tau$-torsionfree left $R$-module then $R^{i} T_{\tau}(M)=0$ for all $i \neq 1$.

Proof. This is a direct consequence of Proposition 1.8 and Proposition 1.11.

(1.13) Proposition. The following conditions on a stable torsion theory $\tau \in R$-tors are equivalent:

(1) $\tau$ is exact;

(2) $R^{i} T_{\tau}(M)=0$ for any left $R$-module $M$ and for all $i>1$;

(3) $R^{2} T_{\tau}(M)=0$ for any left $R$-module $M$.

Proof. (1) $\Rightarrow$ (2): Let $M$ be a left $R$-module. Set $M^{\prime}=T_{\tau}(M)$ and $M^{\prime \prime}=M / M^{\prime}$. Then we have a long exact sequence

$$
\begin{aligned}
0 & \rightarrow R^{0} T_{\tau}\left(M^{\prime}\right) \rightarrow R^{0} T_{\tau}(M) \rightarrow R^{0} T_{\tau}\left(M^{\prime \prime}\right) \rightarrow R^{1} T_{\tau}\left(M^{\prime}\right) \\
& \rightarrow R^{1} T_{\tau}(M) \rightarrow R^{1} T_{\tau}\left(M^{\prime \prime}\right) \rightarrow R^{2} T_{\tau}\left(M^{\prime}\right) \rightarrow \cdots
\end{aligned}
$$

By Proposition 1.4 we see that $R^{i} T_{\tau}\left(M^{\prime}\right)=0$ for all $i>0$ and by Corollary 1.12 we see that $R^{i} T_{\tau}\left(M^{\prime \prime}\right)=0$ for all $i \neq 1$. Therefore, by exactness, $R^{i} T_{\tau}(M)=0$ for all $i>1$.

(2) $\Rightarrow(3)$ : This implication is trivial.

(3) $\Rightarrow$ (1): Let $M$ be $\tau$-torsionfree and $\tau$-injective left $R$-module. Then $E(M)$ is $\tau$-torsionfree and the short exact sequence

$$
0 \rightarrow M \rightarrow E(M) \rightarrow E(M) / M \rightarrow 0
$$


induces a long exact sequence

$$
\begin{aligned}
\cdots & \rightarrow R^{0} T_{\tau}(E(M) / M) \rightarrow R^{1} T_{\tau}(M) \rightarrow R^{1} T_{\tau}(E(M)) \\
& \rightarrow R^{1} T_{\tau}(E(M) / M) \rightarrow R^{2} T_{\tau}(M) \rightarrow \cdots,
\end{aligned}
$$

where $R^{0} T_{\tau}(E(M) / M)=0$ since $E(M) / M$ is $\tau$-torsionfree by Proposition 5.1 of [8] and where $R^{2} T_{\tau}(M)=0$ by (3). Moreover, by Proposition 1.6 we see that $R^{1} T_{\tau}(E(M)) \cong Q_{\tau}(E(M)) / E(M)=0$. Therefore $R^{1} T_{\tau}(E(M) / M)=0$. By Proposition 1.6 this implies that $E(M) / M$ is $\tau$-torsionfree and $\tau$-injective, which establishes (1) by Proposition 16.1 of [8].

(1.14) EXAMPLE. Let $I$ be an ideal of a ring $R$ which is finitely-generated as a left ideal of $R$. Then a left $R$-module $M$ is $\xi(R / I)$-torsion if and only if every element of $M$ is annihilated by a power of $I$. Therefore, in this situation, we see that $R^{n} T_{\xi(R / I)}(M)$ is naturally isomorphic, as an abelian group, to $\lim _{k \geqslant 0} \operatorname{Ext}_{R}^{n}\left(R / I^{k}, M\right)$ for any nonnegative integer $n$. This shows that, in the case of commutative noetherian rings, the functors $R^{n} T_{\xi(R / I)}(-)$ coincide with the local cohomology functors studied by Sharp [18]. In the noncommutative noetherian case we obtain the local cohomology functors studied by Barou [3].

If $I$ is an ideal of a left noetherian ring $R$ then the torsion theory $\xi(R / I)$ is stable if and only if $I$ has the Artin-Rees property with respect to every finitely-generated left $R$-module. That is to say, $\xi(R / I)$ is stable if and only if for every submodule $N$ of a finitely-generated left $R$-module $M$ and for each natural number $n$ there exists a natural number $h=h(n)$ for which $I^{h} M \cap N \subseteq I^{n} N$. [4] This holds, for example, if $R$ is a noetherian ring and if $I$ is generated by a centralizing family of elements (that is, if there exist elements $r_{1}, \ldots, r_{m}$ of $I$ such that the image of each $r_{i}$ is in the center of $R$ modulo the ideal generated by $\left.r_{1}, \ldots, r_{i-1}\right)[3]$.

\section{Various dimensions}

Let $\tau \in R$-tors and let $M$ be a left $R$-module. We define the $\tau$-dimension of $M$, denoted by $\operatorname{dim}_{\tau}(M)$, as follows:

(1) If $\operatorname{supp}(M) \cap \mathbf{P}(\tau)=\varnothing \operatorname{set} \operatorname{dim}_{\tau}(M)=-1$;

(2) If $n$ is a nonnegative integer satisfying the following conditions:

(i) There exists a chain of the form $\pi_{n}<\cdots<\pi_{0}$ in $\mathbf{P}(\tau)$ with $\pi_{0} \in$ $\operatorname{supp}(M) ;$ and

(ii) if $h>n$ there exists no chain of the form $\pi_{h}<\cdots<\pi_{0}$ in $\mathbf{P}(\tau)$ with $\pi_{0} \in \operatorname{supp}(M)$,

then set $\operatorname{dim}_{\tau}(M)=n$; 
(3) otherwise, set $\operatorname{dim}_{\tau}(M)=\infty$.

If $U$ is a nonempty subset of $R$-tors we define $\operatorname{dim}_{U}(M)$ to be $\sup \left\{\operatorname{dim}_{\tau}(M) \mid \tau\right.$ $\in U\}$.

A ring $R$ is left stable if and only if every element of $R$-tors is stable. Left stable left noetherian rings behave very nicely in many ways and they are a convenient generalization of commutative noetherian rings. It is therefore natural to look at them in order to try and calculate that $\tau$-dimension of modules.

Let us recall a construction used in Chapter 12 of [11]. If $\tau \in R$-tors we can define an ascending chain $\tau_{0} \leqslant \tau_{1} \leqslant \cdots$ in $R$-tors, called the Gabriel filtration of $\tau$, by setting $\tau_{0}=\tau$ and $\tau_{i}=\tau_{i-1} \vee\left(\vee\left\{\xi(M) \mid M\right.\right.$ is $\tau_{i-1}$-cocritical $\left.\}\right)$ for all positive integers $i$.

(2.1) Proposition. Let $R$ be a left stable left noetherian ring and let $\tau \in R$-tors. For a $\tau$-torsionfree cocritical left $R$-module $N$ and for a positive integer $i$ the following conditions are equivalent:

(1) $\xi(N) \leqslant \tau_{i}$;

(2) If $\pi_{h}<\cdots<\pi_{0}=\chi(N)$ is a chain in $\mathbf{P}(\tau)$ then $h<i$.

Proof. We will proceed by induction on $i$. First let us consider the case of $i=1$.

Assume (1). Since $N$ is $\tau$-torsionfree and $\tau_{1}$-torsion, there must exist a $\tau$-cocritical left $R$-module $M$ such that $N$ is not $\xi(M)$-torsionfree. By stability, this implies that $N$ is $\xi(M)$-torsion and so there exists a nonzero $R$-homomorphism $\alpha$ from a submodule $M^{\prime}$ of $M$ to $N$. Since $N$ is $\tau$-torsionfree, the map $\alpha$ must be monic. Since $N$ is uniform, this implies that $M^{\prime}$ is isomorphic to a large submodule of $N$ and so $\chi(N)=\chi\left(M^{\prime} \alpha\right)=\chi\left(M^{\prime}\right)=\chi(M)$. Thus, by Proposition 2.5.16 of [17] we see that $\chi(N)$ is a minimal element of $\mathbf{P}(\tau)$, proving (2). Conversely, assume (2). If $\chi(N)$ is a minimal element of $\mathbf{P}(\tau)$ then by Proposition 2.5.16 of [17] there exists a $\tau$-cocritical left $R$-module $M$ satisfying $\chi(N)=\chi(M)$. Hence $N$ is isomorphic to a submodule of $E(M)$ which, by the definition of $\tau_{1}$ and by stability, is $\tau_{1}$-torsion. This proves (1).

Now assume that $i>1$ and that for any $j<i$ we have already established the equivalence of (1) and (2).

Assume that $N$ satisfies (1). If $\xi(N) \leqslant \tau_{i-1}$ then (2) follows by the induction hypothesis. Therefore we can assume that $N$ is not $\tau_{i-1}$-torsion. By stability, this implies that is is $\tau_{i-1}$-torsionfree. As in the proof of the case $i=1$, this implies that $\chi(N)$ is a minimal element of $\mathbf{P}\left(\tau_{i-1}\right)$. Therefore, without loss of generality, we can assume that $N$ is in fact $\pi_{i-1}$-cocritical. If $\chi(N)=\pi_{0}>\cdots>\pi_{h}$ is a chain of torsion theories in $\mathbf{P}(\tau)$ then, by stability, $\pi_{1}$ is of the form $\chi\left(N^{\prime}\right)$, where $N^{\prime}$ is a proper homomorphic image of a submodule of $N$. In particular, $N^{\prime}$ is $\tau_{i-1}$-torsion 
and so, by the induction hypothesis, $h \leqslant i-1$. This proves (2). Conversely, assume (2). If there is no chain in $\mathbf{P}(\tau)$ of the form

$$
\pi_{i-1}<\cdots<\pi_{0}=\chi(N)
$$

then (1) follows by the induction hypothesis. Assume therefore that such a chain exists. Let $N^{\prime}$ be a proper homomorphic image of $N$. If $M$ is a cocritical submodule of $N^{\prime}$ then $\chi(N)>\chi(M)$. Therefore, if $\pi_{h}<\cdots<\pi_{0}=\chi(M)$ is a chain of torsion theories in $\mathbf{P}(\tau)$ we must have $h<i-1$. By the induction hypothesis, this means that $M$ is $\tau_{i-1}$-torsion and so $N^{\prime}$ is $\tau_{i-1}$-torsion. Hence $N$ is either $\tau_{i-1}$-torsion or $\tau_{i-1}$-cocritical. In either case, (1) follows.

(2.2) Proposition. If $R$ is a left stable left noetherian ring and if $\tau \in R$-tors then for a left $R$-module $M$ and for a nonnegative integer $n$ the following conditions are equivalent:

(1) $\xi(M) \leqslant \tau_{n+1}$ and $\xi(M) \neq \tau_{n}$.

(2) $\operatorname{dim}_{\tau}(M)=n$.

Proof. (1) $\Rightarrow(2)$ : By (1), $M$ is not $\tau_{n}$-torsion and so there exists a cocritical submodule $N$ of $M$ which is not $\tau_{n}$-torsion and hence is $\tau_{n}$-torsionfree. On the other hand, $M$ is $\tau_{n+1}$-torsion and hence so is $N$. Thus, by Proposition 2.1, $\chi(N) \in \operatorname{supp}(M)$ and there exists a chain of the form $\pi_{n}<\cdots<\pi_{0}=\chi(N)$ in $\mathbf{P}(\tau)$. This proves that $\operatorname{dim}_{\tau}(M) \geqslant n$. Now assume that there exists an element $\pi$ of $\operatorname{supp}(M)$ and a chain $\pi_{h}^{\prime}<\cdots<\pi_{0}^{\prime}=\pi$ in $\mathbf{P}(\tau)$ with $h>n$. Then $M$ is not $\pi$-torsion and so there exists a cocritical submodule $N^{\prime}$ of $M$ which is not $\pi$-torsion and hence is $\pi$-torsionfree. This implies that $\chi\left(N^{\prime}\right) \geqslant \pi$. By Proposition 2.1 , this implies that $N^{\prime}$ is not $\tau_{n+1}$-torsion, and so neither is $M$. This contradicts (1), proving (2).

(2) $\Rightarrow$ (1): From (2) we deduce that if $N$ is a cocritical submodule of $M$ then $N$ is either $\tau$-torsion or for any chain $\pi_{h}<\cdots<\pi_{0}=\chi(N)$ in $\mathbf{P}(\tau)$ we have $h<n+1$. Therefore, by Proposition 2.1 we see that every such module $N$ is $\tau_{n+1}$-torsion. By stability, this implies that $M$ is $\tau_{n+1}$-torsion and so $\xi(M) \leqslant \tau_{n+1}$. On the other hand, there exists an element $\pi$ of $\operatorname{supp}(M)$ and a chain $\pi_{n}<\cdots<$ $\pi_{0}=\pi$. Since $M$ is not $\pi$-torsion, there exists a cocritical submodule $N^{\prime}$ of $M$ which is not $\pi$-torsion and hence is $\pi$-torsionfree. Therefore $\chi\left(N^{\prime}\right) \geqslant \pi$. Indeed, by the condition on the lengths of chains we must in fact have equality here. By Proposition 2.1 , this means that $\xi\left(N^{\prime}\right) \$ \tau_{n}$ and so $\xi(M) \$ \tau_{n}$.

We will say that a ring $R$ is left effective if and only if it is left stable, left noetherian, and every element of $R$-sp is exact. By Proposition 17.1 of [8] we see that, in the presence of the noetherian condition, this last condition is equivalent to the condition that every element of $R$-sp is perfect. Commutative noetherian 
rings are clearly left effective. By Example 6.16 of [12] and by Proposition 9 of [22] we see that left noetherian Azumaya algebras are left effective.

(2.3) EXAMPLE. Let $R$ be a prime hereditary noetherian quasi-local ring which is a bounded order in its classical ring of fractions. We claim that $R$ is left effective. Indeed, since $R$ is left hereditary, we know that every element of $R$-tors is exact by Proposition 16.4 of [8]. Moreover, by Proposition IV.1.7 of [15] we see that $R$ is fully left bounded and left noetherian so the map $P \mapsto \chi(R / P)$ is a bijective correspondence between the set $\operatorname{spec}(R)$ of all prime ideals of $R$ and $R$-sp. See Propositions 6.7 and 6.11 of [11] for details. By Proposition IV.1.1 of [15] we see that the Goldie torsion theory in $R$-tors is faithful and so it equals the Lambek torsion theory $\chi(R)$. Therefore $\chi(R)$ is stable. If $R$ is not simple then by the quasi-locality of $R$ we see that the Jacobson radical $J(R)$ is the only nonzero prime ideal of $R$ and that $\chi(R / J(R))=\xi$, since any nonzero ideal of $R$ is a power of $J(R)$. (See pages 50-51 of [15].) Therefore $\chi(R / J(R))$ is also stable, proving that $R$ is left stable and so left effective. Examples of rings of this type can be found in sections I.8 and III.4 of [15].

(2.4) Proposition. Let $R$ be a left effective ring and let $\tau \in R$-tors. If $M$ is a left $R-$ module and if $i$ is a natural number satisfying $R^{i} T_{\tau}(M) \neq 0$ then $i \leqslant \operatorname{dim}_{\tau}(M)+$ 1 .

Proof. Set $k=\operatorname{dim}_{\tau}(M)$. If $k=\infty$ the result is trivial so we may assume that $k$ is finite. If $k=-1$ the result follows from Proposition 1.4 and so we may assume that $k$ is nonnegative. Since $M$ is the direct union of the directed system of its finitely-generated submodules, it suffices to show that $R^{i} T_{\tau}\left(M^{\prime}\right)=0$ for all $i>k+1$ and for any finitely-generated submodule $M^{\prime}$ of $M$. Thus, without loss of generality, we can assume that $M$ itself is finitely-generated and hence noetherian. Since $R$ is left noetherian, it is surely left definite and so every nonzero homomorphic image of $M$ has a nonzero cocritical submodule. Since $M$ is assumed to be noetherian, this means that we can find a chain

$$
0=N_{0} \subset N_{1} \subset \cdots \subset N_{u}=M
$$

of submodules of $M$ satisfying the condition that $\bar{N}_{h}=N_{h} / N_{h-1}$ is cocritical for all $1 \leqslant h \leqslant u$. To prove the proposition, it suffices to show that $R^{i} T_{\tau}\left(\bar{N}_{h}\right)=0$ for all $1 \leqslant h \leqslant u$ and all $i>k+1$. To do this, we proceed by induction on $k$.

First assume that $k=0$. If $\bar{N}_{h}$ is $\tau$-torsion the desired result follows from Proposition 1.4. Therefore assume that it is not $\tau$-torsion. By stability, this implies that $\bar{N}_{h}$ is $\tau$-torsionfree and so $\pi_{h}=\chi\left(\bar{N}_{h}\right) \in \mathbf{P}(\tau)$. By Proposition 1.11, we know that $R^{i} T_{\pi_{h}}\left(E_{\pi_{h}}\left(\bar{N}_{h}\right)\right)=0$ for all $i \geqslant 0$. By Proposition 1.10, this implies that $R^{i} T_{\tau}\left(E_{\pi_{h}}\left(\bar{N}_{h}\right)\right)=0$ for all $i \geqslant 0$. Set $N_{h}^{\prime}=E_{\pi_{h}}\left(\bar{N}_{h}\right) / \bar{N}_{h}$. We claim that $N_{h}^{\prime}$ is 
$\tau$-torsion. Indeed, if $\pi \in \operatorname{supp}\left(N_{h}^{\prime}\right) \cap \mathbf{P}(\tau)$ then $\pi \in \operatorname{supp}\left(E_{\pi_{h}}\left(\bar{N}_{h}\right)\right)=\operatorname{supp}\left(\bar{N}_{h}\right)$ so, by stability and by the uniformity of $\bar{N}_{h}$, we see that $\bar{N}_{h}$ must be $\pi$-torsionfree. Therefore $\pi_{h}=\chi\left(\bar{N}_{h}\right) \geqslant \pi$. Since $N_{h}^{\prime}$ is in fact $\pi_{h}$-torsion by construction, this inequality must be strict. But this is a contradiction for, by construction, $\pi_{h}$ is a minimal element of $\mathbf{P}(\tau) \cap \operatorname{supp}(M)$. Therefore $\pi \notin \mathbf{P}(\tau)$. Thus we see that $N_{h}^{\prime}$ is $\pi$-torsion for all $\pi \in \mathbf{P}(\tau)$ and so $N_{h}^{\prime}$ is $\tau$-torsion, as claimed.

The short exact sequence $0 \rightarrow \bar{N}_{h} \rightarrow E_{\pi_{h}}\left(\bar{N}_{h}\right) \rightarrow N_{h}^{\prime} \rightarrow 0$ induces a long exact sequence

$$
\begin{aligned}
0 & \rightarrow R^{0} T_{\tau}\left(\bar{N}_{h}\right) \rightarrow R^{0} T_{\tau}\left(E_{\pi_{h}}\left(\bar{N}_{h}\right)\right) \rightarrow R^{0} T_{\tau}\left(N_{h}^{\prime}\right) \rightarrow R^{1} T_{\tau}\left(\bar{N}_{h}\right) \\
& \rightarrow R^{1} T_{\tau}\left(E_{\pi_{h}}\left(\bar{N}_{h}\right)\right) \rightarrow R^{1} T_{\tau}\left(N_{h}^{\prime}\right) \rightarrow R^{2} T_{\tau}\left(\bar{N}_{h}\right) \rightarrow \cdots
\end{aligned}
$$

with respect to which we note the following:

(1) $R^{0} T_{\tau}\left(\bar{N}_{h}\right)=R^{0} T_{\tau}\left(E_{\pi_{h}}\left(\bar{N}_{h}\right)\right)=0$ by $\tau$-torsionfreeness;

(2) $R^{i} T_{\tau}\left(E_{\pi_{h}}\left(\bar{N}_{h}\right)\right)=0$ for all $i \geqslant 0$, as remarked above;

(3) $N_{h}^{\prime}$ is $\tau$-torsion by the above claim and so $R^{i} T_{\tau}\left(N_{h}^{\prime}\right)=0$ for all $i>0$ by Proposition 1.4.

Therefore, by exactness, $R^{i} T_{\tau}\left(\bar{N}_{h}\right)=0$ for all $i>1$, which is what we wanted to show.

Now assume that $k>0$ and that for any left $R$-module $M^{\prime \prime}$ satisfying $\operatorname{dim}_{\tau}\left(M^{\prime \prime}\right)<k$ we have $R^{i} T_{\tau}\left(M^{\prime \prime}\right)=0$ for all $i>\operatorname{dim}_{\tau}\left(M^{\prime \prime}\right)+1$. In particular, we know that $R^{i} T_{\tau}\left(\bar{N}_{h}\right)=0$ whenever $i>k+1$ and $\operatorname{dim}\left(\bar{N}_{h}\right)<k$ so we need consider only those indices $h$ for which $\operatorname{dim}_{\tau}\left(\bar{N}_{h}\right)=k$. Moreover, as before, we can assume that $\bar{N}_{h}$ is $\tau$-torsionfree.

We claim that in this situation $N_{h}^{\prime}=E_{\pi_{h}}\left(\bar{N}_{h}\right) / \bar{N}_{h}$ satisfies $\operatorname{dim}_{\tau}\left(N_{h}^{\prime}\right)<k$. Indeed, since $\operatorname{dim}_{\tau}\left(\bar{N}_{h}\right)=k$ we see that $\bar{N}_{h}$ is $\delta(U)$-torsion, where $U$ is the subset of $\mathbf{P}(\tau)$ consisting of those elements $\pi^{\prime}$ for which any chain of the form $\pi_{t}<\cdots<$ $\pi_{0}=\pi^{\prime}$ in $\mathbf{P}(\tau)$ satisfies $t \leqslant k$. By stability, $E_{\pi_{h}}\left(\bar{N}_{h}\right)$ is also $\delta(U)$-torsion and hence so is $N_{h}^{\prime}$. This is equivalent to the condition that $\varnothing \neq \operatorname{ass}\left(N_{h}^{\prime} / N\right) \subseteq U$ for every proper submodule $N$ of $N_{h}^{\prime}$. But for each such $N$ we have ass $\left(N_{h}^{\prime} / N\right) \subseteq$ $\operatorname{supp}\left(N_{h}^{\prime} / N\right) \subseteq \operatorname{supp}\left(N_{h}^{\prime}\right) \subseteq \operatorname{supp}\left(E_{\pi_{h}}\left(\bar{N}_{h}\right)\right)=\left\{\pi^{\prime} \in R-\operatorname{sp} \mid \pi^{\prime} \leqslant \pi_{h}\right\}$. Thus if $\pi \in$ ass $\left(N_{h}^{\prime} / N\right)$ we have $\pi \leqslant \pi_{h}$ and in fact we cannot have equality here since $N_{h}^{\prime}$ is $\pi_{h}$-torsion but not $\pi$-torsion.

Let $U^{\prime}$ be the set of those elements $\pi^{\prime}$ in $U$ for which there is no chain of the form $\pi_{k}<\cdots<\pi_{0}=\pi^{\prime}$ in $\mathbf{P}(\tau)$. Since $\pi_{h} \in U$, we see by the above that $\pi \in U^{\prime}$. Thus for any proper submodule $N$ of $N_{h}^{\prime}$ we have $\varnothing \neq \operatorname{ass}\left(N_{h}^{\prime} / N\right) \subseteq U^{\prime}$. This shows that $N_{h}^{\prime}$ is $\delta\left(U^{\prime}\right)$-torsion and so $\operatorname{dim}_{\tau}\left(N_{h}^{\prime}\right)<k$, as claimed. By the induction hypothesis, this means that $R^{i} T_{\tau}\left(N_{h}^{\prime}\right)=0$ for all $i>k$. Again, as before, $R^{i} T_{\tau}\left(E_{\pi_{h}}\left(\bar{N}_{h}\right)\right)=0$ for all $i \geqslant 0$ and so from the long exact sequence

$$
\cdots \rightarrow R^{i} T_{\tau}\left(N_{h}^{\prime}\right) \rightarrow R^{i+1} T_{\tau}\left(\bar{N}_{h}\right) \rightarrow R^{i+1} T_{\tau}\left(E_{\pi_{h}}\left(\bar{N}_{h}\right)\right) \rightarrow \cdots
$$

we deduce that $R^{i} T_{\tau}\left(\bar{N}_{h}\right)=0$ for all $i>k+1$. 
(2.5) Proposition. Let $R$ be a left effective ring and let $\tau \in R$-tors. If $M$ is a left $R$-module and if $i$ is a natural number satisfying $R^{i} T_{\tau}(M) \neq 0$ then $i \leqslant \operatorname{dim}_{\mathrm{V}(\tau)}(M)$.

Proof. Set $k=\operatorname{dim}_{\mathbf{v}_{(\tau)}}(M)$. If $k$ is infinite then we are done trivially and so we can assume that $k$ is finite. Assume $k=-1$. Then $\operatorname{supp}(M) \subseteq \mathbf{P}(\tau)$. If

$$
0 \rightarrow M \rightarrow E_{0} \stackrel{\alpha_{0}}{\rightarrow} E_{1} \stackrel{\alpha_{1}}{\rightarrow} E_{2} \rightarrow \cdots
$$

is a minimal injective resolution of $M$ then for all $i \geqslant 0$ we have $\operatorname{supp}\left(E_{i}\right) \subseteq$ $\operatorname{supp}(M) \subseteq \mathbf{P}(\tau)$. The ring $R$ is left noetherian and so, in particular, left definite. Therefore each $E_{i}$ is $\tau$-torsionfree and so $R^{i} T_{\tau}(M)=0$ for all $i \geqslant 0$.

We are left to consider the case of $k$ nonnegative. As in the proof of Proposition 2.4, we can assume without loss of generality that $M$ is a noetherian left $R$-module. There therefore exists a chain

$$
0=N_{0} \subset N_{1} \subset \cdots \subset N_{u}=M
$$

of submodules of $M$ satisfying the condition that $\bar{N}_{h}=N_{h} / N_{h-1}$ is cocritical for all $1 \leqslant h \leqslant u$. To prove the proposition, it therefore suffices to show that $R^{i} T_{\tau}\left(\bar{N}_{h}\right)=0$ for all $1 \leqslant h \leqslant u$ and all $i>k$. To do this, we proceed by induction on $k$.

Assume that $k=0$ and let $1 \leqslant h \leqslant u$. If $\bar{N}_{h}$ is not $\tau$-torsionfree then it is $\tau$-torsion and we are done by Proposition 1.4. Therefore assume that $\bar{N}_{h}$ is $\tau$-torsionfree and consider a minimal injective resolution

$$
0 \rightarrow \bar{N}_{h} \rightarrow E_{0} \stackrel{\alpha_{0}}{\rightarrow} E_{1} \stackrel{\alpha_{1}}{\rightarrow} E_{2} \rightarrow \cdots
$$

of $\bar{N}_{h}$. To show that $R^{i} T_{\tau}\left(\bar{N}_{h}\right)=0$ for all $i>0$ it suffices to show that $E_{i}$ is $\tau$-torsionfree for each $i>0$. Set $\pi_{h}=\chi\left(\bar{N}_{h}\right)$.

We first note that $\operatorname{supp}\left(\bar{N}_{h}\right) \subseteq \mathbf{P}(\tau)$. Indeed, if this were not the case then there would exist a torsion theory $\pi$ belonging to $\operatorname{supp}\left(\bar{N}_{h}\right) \cap \mathbf{V}(\tau)$. Since $\bar{N}_{h}$ is not $\pi$-torsion, it is $\pi$-torsionfree and so $\pi<\pi_{h}$, contradicting the assumption that $\operatorname{dim}_{\pi}(M) \leqslant \operatorname{dim}_{\mathrm{v}(\tau)}(M)=0$. We next note that by Proposition 2.7.16, 2.7.4, and 2.6.1 of [17] we have supp $\left(\bar{N}_{h}\right)=\left\{\pi \in R\right.$-sp $\left.\mid \pi^{\prime} \leqslant \pi_{h}\right\}$. Thus, in particular, we see that $\operatorname{supp}\left(E_{0}\right)=\operatorname{supp}\left(\bar{N}_{h}\right)$ and if $i>0$ then $\operatorname{supp}\left(E_{i}\right)=\operatorname{supp}\left(E_{i-1} / \operatorname{ker}\left(\alpha_{i-1}\right)\right) \subseteq$ $\operatorname{supp}\left(E_{i-1}\right)$ and so $\operatorname{supp}\left(E_{i}\right) \subseteq \operatorname{supp}\left(\bar{N}_{h}\right) \subseteq \mathbf{P}(\tau)$ for all $i \geqslant 0$. Since $R$ is left noetherian and so, in particular, left definite, this implies that each $E_{i}$ is $\tau$-torsionfree and so $R^{i} T_{\tau}\left(\bar{N}_{h}\right)=0$ for all $i>0$.

Now assume that $k>0$ and that for any left $R$-module $M^{\prime \prime}$ satisfying $\operatorname{dim}_{\mathrm{V}(\tau)}\left(M^{\prime \prime}\right)<k$ we have $R^{i} T_{\tau}\left(M^{\prime \prime}\right)=0$ for all $i>\operatorname{dim}_{\mathrm{V}(\tau)}\left(M^{\prime \prime}\right)$. In particular, 
we know that $R^{i} T_{\tau}\left(\bar{N}_{h}\right)=0$ whenever $i>k$ and $\operatorname{dim}_{\mathbf{v}(\tau)}\left(\bar{N}_{h}\right)<k$. Assume therefore that $1 \leqslant h \leqslant u$ satisfies $\operatorname{dim}_{\mathrm{V}(\tau)}\left(\bar{N}_{h}\right)=k$. If $\bar{N}_{h}$ is $\tau$-torsion the desired result follows from Proposition 1.4. Hence we can assume without loss of generality that $\bar{N}_{h}$ is $\tau$-torsionfree.

Consider the exact sequence of left $R$-modules

$$
0 \rightarrow \bar{N}_{h} \rightarrow E_{\pi_{h}}\left(\bar{N}_{n}\right) \rightarrow N_{h}^{\prime} \rightarrow 0
$$

in which $N_{n}^{\prime}=E_{\pi_{h}}\left(\bar{N}_{h}\right) / \bar{N}_{h}$. Note that $\operatorname{supp}\left(N_{h}^{\prime}\right) \subseteq \operatorname{supp}\left(E_{\pi_{h}}\left(\bar{N}_{h}\right)\right)=\operatorname{supp}\left(\bar{N}_{h}\right)=$ $\left\{\pi^{\prime} \in R\right.$-sp $\left.\mid \pi^{\prime} \leqslant \pi_{h}\right\}$. Moreover, $N_{h}^{\prime}$ is $\pi_{h}$-torsion so if $\pi \in \mathbf{V}(\tau) \cap \operatorname{supp}\left(N_{h}^{\prime}\right)$ then $\operatorname{dim}_{\pi}\left(N_{h}^{\prime}\right)<\operatorname{dim}_{\pi}\left(\bar{N}_{h}\right) \leqslant k$ whence $\operatorname{dim}_{\mathbf{v}_{(\tau)}}\left(N_{h}^{\prime}\right)<k$. Hence, by the induction hypothesis, we see that $R^{i} T_{\tau}\left(N_{h}^{\prime}\right)=0$ for all $i \geqslant k$. But the short exact sequence (*) induces a long exact sequence

$$
\begin{aligned}
\cdots & \rightarrow R^{i} T_{\tau}\left(E_{\pi_{h}}\left(\bar{N}_{h}\right)\right) \rightarrow R^{i} T_{\tau}\left(N_{h}^{\prime}\right) \rightarrow R^{i+1} T_{\tau}\left(\bar{N}_{h}\right) \\
& \rightarrow R^{i+1} T_{\tau}\left(E_{\pi_{h}}\left(\bar{N}_{h}\right)\right) \rightarrow \cdots
\end{aligned}
$$

Since $R^{i} T_{\tau}\left(E_{\pi_{h}}\left(\bar{N}_{h}\right)\right)=0$ for all $i \geqslant 0$ by Proposition 1.11 and since $R^{i} T_{\tau}\left(N_{h}^{\prime}\right)=0$ for all $i \geqslant k$, this implies that $R^{i} T_{\tau}\left(\bar{N}_{h}\right)=0$ for all $i>k$, which is what we needed to prove.

Note that, for any left $R$-module $M, \operatorname{dim}_{\xi}(M)$ is precisely the torsion-theoretic Krull dimension (or TTK-dimension) of $M$ as introduced in [9] and [13] and developed in detail in [11]. By Proposition 13.3 of [11] we see that if $R$ is left effective then this coincides with the Gabriel dimension of $M$. Moreover, it is clear from the definitions that $\operatorname{dim}_{U}(M) \leqslant \operatorname{dim}_{\xi}(M)$ for any nonempty subset $U$ of $R$-tors. We therefore obtain the following immediate corollary of the previous result.

(2.6) Corollary. Let $R$ be a left effective ring and let $\tau \in R$-tors. If $M$ is a left $R$-module and if $i$ is a natural number satisfying $R^{i} T_{\tau}(M) \neq 0$ then $i$ is no greater than the Gabriel dimension of $M$.

\section{Acknowledgement}

Portions of this paper were prepared while the second author was visiting the University of Haifa under the auspices of the Scientific Exchange Agreement between the governments of France and Israel. 


\section{References}

[1] T. Albu and C. Nastasescu, 'Local cohomology and torsion theory. I', Rev. Roumaine Math. Pures Appl. 26 (1981), 3-14.

[2] T. Albu and C. Nastasescu, 'Some aspects of non-noetherian local cohomology', Comm. Algebra 8 (1980), 1539-1560.

[3] G. Barou, 'Cohomologie locale des algèbres enveloppants d'algèbres de Lie nilpotentes', Séminaire d'Algèbre Paul Dubreil, edited by M. Malliavin (Lecture Notes in Mathematics 641, Springer-Verlag, Berlin, 1978).

[4] J. Beachy, 'On the torsion theoretic support of a module', Hokkaido Math. J. 6 (1977), 16-27.

[5] M. H. Bijan-Zadeh, 'Torsion theories and local cohomology over commutative noetherian rings', J. London Math. Soc. (2) 19 (1979), 402-410.

[6] P.-J. Cahen, 'Commutative torsion theory', Trans. Amer. Math. Soc. 184 (1973), 73-85.

[7] S. Dickson, 'Direct decompositions of radicals', Proceedings of the conference on categorical algebra, La Jolla, 1965 (Springer-Verlag, Berlin, 1966).

[8] J. S. Golan, Localization of noncommutative rings (Marcel Dekker, New York, 1975).

[9] J. S. Golan, 'A Krull-like dimension for noncommutative rings', Israel J. Math. 19 (1974), 297-304.

[10] J. S. Golan, 'A characterization of left semiartinian rings', Bull. Austral. Math. Soc. 11 (1974), $425-428$.

[11] J. S. Golan, Decomposition and dimension in module categories (Marcel Dekker, New York, 1977).

[12] J. S. Golan, Structure sheaves over a noncommutative ring (Marcel Dekker, New York, 1980).

[13] J. S. Golan and J. Raynaud, 'Dimension de Gabriel et TTK-dimension de modules', $C$. $R$. Acad. Sci. Paris 278 (1974), A1603-Al606.

[14] T. Kato, 'Rings of $U$-dominant dimension $\geqslant 1$ ', Tôhoku Math. J. 21 (1969), 321-327.

[15] G. Maury and J. Raynaud, Ordres maximaux au sens de $K$. Asano (Lecture Notes in Mathematics 808, Springer-Verlag, Berlin, 1980).

[16] R. W. Miller, 'Finitely generated projective modules and TTF classes', Pacific J. Math. 64 (1976), 505-515.

[17] J. Raynaud, Localisations et spectres d'anneaux (Docteur d'Etat thesis, Université ClaudeBernard, Lyon, 1976).

[18] R. Y. Sharp, 'Local cohomology theory in commutative algebra', Quart. J. Math. Oxford Ser. (2) 21 (1970), 425-434.

[19] B. Stenström, Rings of quotients (Springer-Verlag, Berlin, 1975).

[20] H. Storrer, 'Torsion theories and dominant dimension', appended to J. Lambek, Torsion theories, additive semantics, and rings of quotients (Lecture Notes in Mathematics 177, Springer-Verlag, Berlin, 1971).

[21] K. Suominen, 'Localization of sheaves and Cousin complexes', Acta Mathematica 131 (1973), 27-41.

[22] F. Van Oystaeyen 'Note on the torsion theory at a prime ideal of a left noetherian ring', $J$. Pure Appl. Algebra 6 (1975), 297-304.

Department of Mathematics

University of Haifa

31999 Haifa

Israel
Département de Mathématiques Université Claude-Bernard (Lyon I) 69622 Villeurbanne Cedex France 